\title{
Ultraviolet Photodetachment Spectroscopy on Jet-cooled Metal-cluster Anions
}

\author{
Gerd Ganteför, Michael Gausa, Karl-Heinz Meiwes-Broer* and Hans O. Lutz \\ Fakultät für Physik, Universität Bielefeld, 4800 Bielefeld 1, Federal Republic of Germany
}

\begin{abstract}
Metal-cluster anions have been produced by laser vaporization and cooled in a supersonic expansion, without converting neutral clusters into anions via a secondary ionizing agent. After mass-selection, photoelectrons were detached $(h \nu=3.68 \mathrm{eV})$ from defined anion bunches and analysed in a magnetic bottle-type time-of-flight electron spectrometer. The resulting photoelectron distributions from $\mathrm{Al}_{n}^{-}, \mathrm{Ni}_{n}^{-}, \mathrm{Ag}_{n}^{-}$and $\mathrm{Sn}_{n}^{-}, n<22$, are compared; all spectra display a dramatic dependence on the cluster size and differ substantially from the respective bulk spectra. The measurements provide information about low-lying excited electronic structures which are missing in the solid metal. Photoelectron thresholds as estimates of electron affinities in part follow a simple electrostatic model.
\end{abstract}

The photoelectric effect was discovered in the last century by Lenard and Thompson; today it is a powerful tool for investigating the electronic behaviour of matter (gas-phase atoms and molecules, pure and covered bulk material and liquids). Photon energies range from a few eV to several $\mathrm{keV}$. Therefore, all electronic levels, starting from the atomic core up to valence levels that contribute to chemical bonds, can be explored by this effect. When measuring the kinetic energy, $E_{\mathrm{kin}}$, of the photoelectron, the difference between the electronic states before and after ionization is roughly given by $h \nu-E_{\text {kin }}$, $h \nu$ being the photon energy. In addition, one has to consider the change of vibrational, rotational and translational energy upon electron emission, which might alter $E_{\text {kin }}$.

This technique has only recently been applied to gas-phase elemental clusters, ${ }^{1-4}$ where mainly visible or u.v. irradiation has been used to cause electron detachment. One of the reasons that cluster studies are attractive is that these systems may provide a link between atoms or molecules and the condensed state. At present much evidence exists for a number of properties of small aggregates being quite different from those of the respective bulk systems. ${ }^{5}$ For example, the spatial symmetry of clusters can differ substantially from that of the bulk crystalline structure; five-fold symmetry axes may often be found in free clusters, ${ }^{6}$ while in solids they are an exception. Furthermore, the chemical reactivity is known to be strongly size-dependent. ${ }^{7-9}$ Photoionization of neutral metal [see, e.g. ref. (10)-(12)], compound ${ }^{13}$ and rare-gas clusters ${ }^{14}$ has widely been investigated; in each case the ionization turned out to be different from the respective process in the solid.

Photoelectron spectroscopy on mass-selected metal-cluster anions as presented in this contribution reveals not only that the electron affinities are strongly size- and material-dependent but, additionally, that the photoelectron spectra are characteristic for a defined cluster. Well resolved bands with often very pronounced gaps are characteristic of each cluster as its size evolves from small to large $n$. A direct comparison of experimental results with calculations, however, depends on the degree to which the theory is applicable to real experimental conditions: some clusters tend to appear in a variety of isomers or 'nearly degenerate' electronic states [e.g. in the case of $\mathrm{Al}_{n}^{-}, c f$. ref. (15)]. Therefore, one prerequisite for clarifying the situation is the experimental investigation of clusters at very low temperatures, thereby possibly obtaining clusters in a 


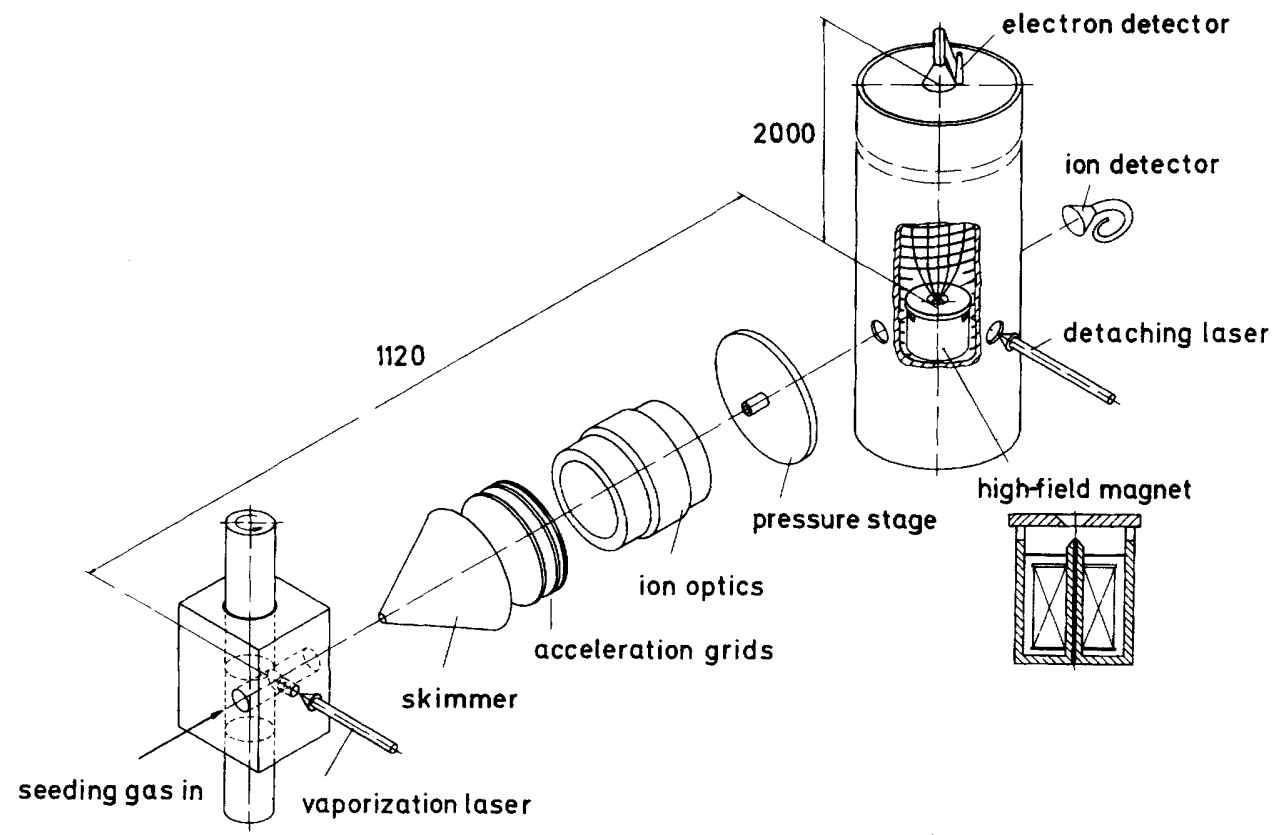

Fig. 1. Schematic diagram of the experimental set-up. Metal-cluster anions are produced by laser vaporization without post-ionization, and cooled by supersonic expansion (left). After pulse formation electrons are photodetached from mass-identified cluster bunches and undergo energy analysis in a magnetic bottle spectrometer (right). The shape of the high-field magnet is shown in the insert. Distances in $\mathrm{nm}$.

defined state. Experiments on jet-cooled anions, as reported here, may be one way to solve the problem.

\section{Experimental}

A schematic diagram of the apparatus is shown in fig. 1. To obtain a high pressure gradient (source operation at $10^{-4} \mathrm{mbar}$, collision-free acceleration of the cluster ions at $10^{-6}$ mbar, photodetachment at $10^{-8}$ mbar) the vacuum system consists of three differentially pumped subunits.

The metal-cluster beam source employs pulsed plasma formation by high-power laser light focussed onto a target rod. Infrared, visible or ultraviolet light are suitable for this process. For the measurements presented in this communication light from an $\mathrm{XeCl}$ excimer laser $(100 \mathrm{~mJ}$ per pulse, $308 \mathrm{~nm}$ ) was used, producing an intense plasma which is flushed by a carrier-gas pulse through a channel and a conical nozzle into high vacuum. ${ }^{16}$ No additional ionization is necessary to generate positive or negative cluster ions of numerous materials. $3,15,17$

The plasma jet emerging from the nozzle without electric acceleration passes a skimmer which serves as a pressure stage. At nearly collision-free conditions the bunches are then separated into charge-defined cluster ions by means of a pulsed acceleration system. Mass spectra are obtained by analysing the drift times in a time-of-flight (TOF) mass spectrometer operating collinearly with the expansion direction.

On their way to the detector the cluster ion-pulse train passes the detachment region of a TOF electron spectrometer. U.v. light (nitrogen laser, $h \nu=3.68 \mathrm{eV}$, pulse length $=$ $10 \mathrm{~ns}$ ) serves to detach electrons from mass-identified anion bunches. The principle of 


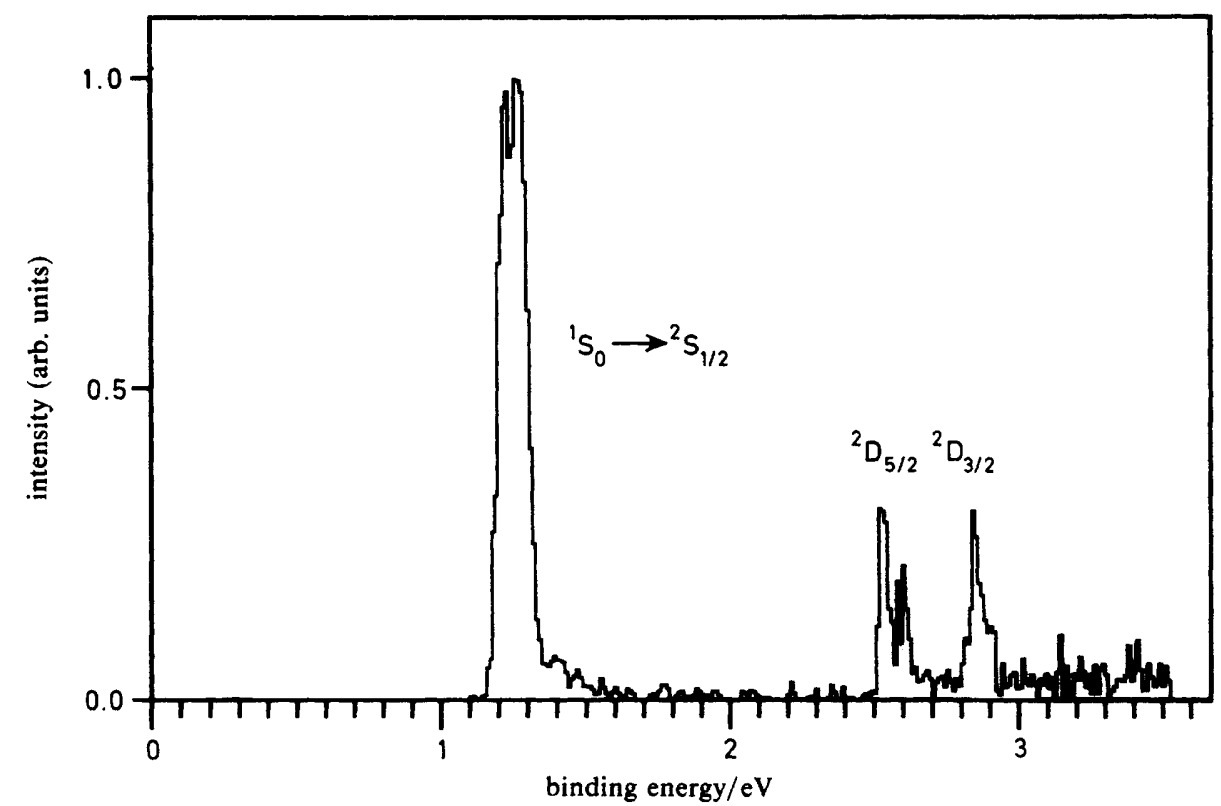

Fig. 2. Photoelectron calibration spectrum of the copper anion $\left(\mathrm{Cu}_{1}^{-}\right)$at a beam energy of $30 \mathrm{eV}$. With a laser photon energy of $h \nu=3.68 \mathrm{eV}$ excited atom states $\left({ }^{2} \mathrm{D}_{5 / 2},{ }^{2} \mathrm{D}_{3 / 2}\right)$ are also accessible and give two further calibration points.

this electron spectrometer is based on an idea of Kruit and Read. ${ }^{18}$ The electrons are accelerated in a strong magnetic field, and subsequently pass adiabatically into a weak-field zone which guides them into the detector. The design and operation of our instrument is described elsewhere. ${ }^{15}$ In contrast with former experiments [ref. (3) and (15)] we used here a stronger electromagnet for the high-field region $(1800 \mathrm{G})$ and a longer drift tube $(2 \mathrm{~m})$. With these modifications the overall electron spectrometer resolution reaches $\Delta E / E=0.05$.

Energy calibration is achieved by recording photoelectron (p.e.) spectra of monomers at different wavelengths, using published electron affinities $\left(A_{\mathrm{e}}\right){ }^{19,20}$ As an example fig. 2 shows a p.e. spectrum of $\mathrm{Cu}^{-}$taken at a photon energy of $h \nu=3.68 \mathrm{eV}$. Such an energy spectrum is obtained by numerical transformation of the TOF spectrum. As is usual, the data are plotted as a function of electron binding energy, given by the photon energy minus the kinetic energy of the detached electron. The vertical axis represents full-scale photoelectron count so that the intensities are not directly related to detachment cross-sections. The first line corresponds to the $\left({ }^{1} \mathrm{~S}_{0} \rightarrow{ }^{2} \mathrm{~S}_{1 / 2}\right)$ ground-state transition; in addition, low-lying excited states of neutral copper $\left({ }^{2} \mathrm{D}_{5 / 2},{ }^{2} \mathrm{D}_{3 / 2}\right)^{21}$ contribute to the spectrum and give two other calibration points. Note that $S \rightarrow D$ transitions are forbidden in optical spectroscopy; since this selection rule is not valid in p.e. spectroscopy the D states are populated with high intensity. The imperfection of the lineshapes is to a large part due to the initial cluster ion's velocity (Doppler broadening); therefore, this effect decreases with increasing ion mass (i.e. cluster size).

\section{Results}

Fig. 3 show as examples $\mathrm{Sn}_{n}^{-}$and $\mathrm{Al}_{n}^{-}$mass spectra. The intensity distributions of all negatively and positively charged metal clusters which we studied so far look amazingly 

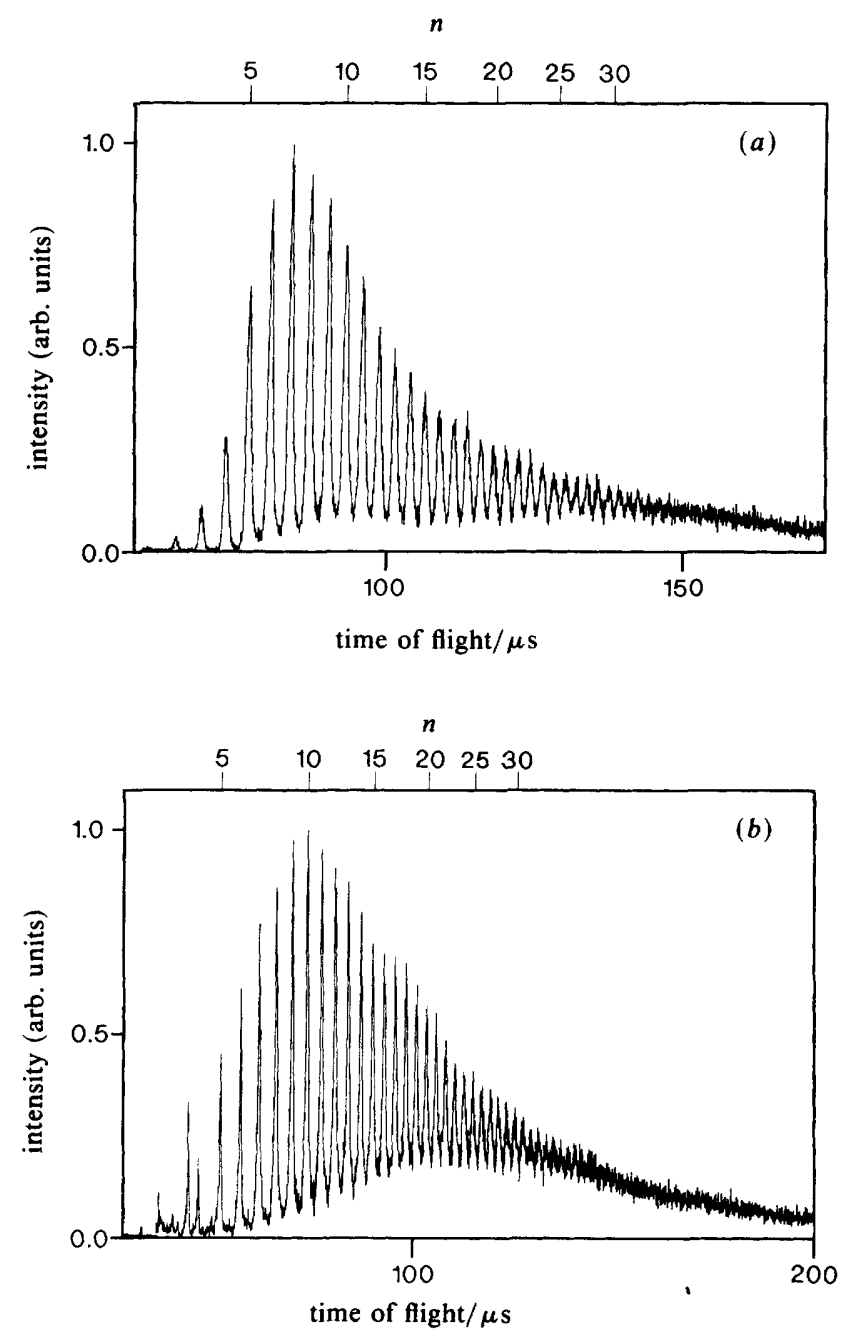

Fig. 3. Time-of-flight mass spectra of tin $(a)$ and aluminium $(b)$ cluster anions, produced by direct laser vaporization (without additional ionization). The ions are analysed collinearly to the source direction; their energy is $300 \mathrm{eV}$.

similar. With the exception of a few contaminant lines mainly pure metal cluster anions are detected. The envelopes of such intensity distributions can be changed by adjusting experimental parameters, but fine structures (e.g. 'magic numbers') are weak or do not appear at all.

Since the TOF cluster mass spectrometer operates collinearly to the source direction, a lineshape analysis at low beam energies can give information about the ion's energy spread, or 'translational temperature', $T_{\text {trans }}$ : a thermal ion velocity distribution before acceleration will (after acceleration under Wiley-McLaren conditions ${ }^{22}$ ) result in an approximately Gaussian mass peak. To first order, its relative width is linearly dependent on the ratio of the thermal velocity to the velocity after acceleration. Therefore, in the case of very low acceleration voltages it is possible to measure $T_{\text {trans }}$ even for cold ions. For $\mathrm{Cu}$, accellerated to $20 \mathrm{eV}$, we find a translational temperature of $c a .6 \mathrm{~K}$. Such a measurement is only possible at low (an-)ion intensities. At higher intensities, which 

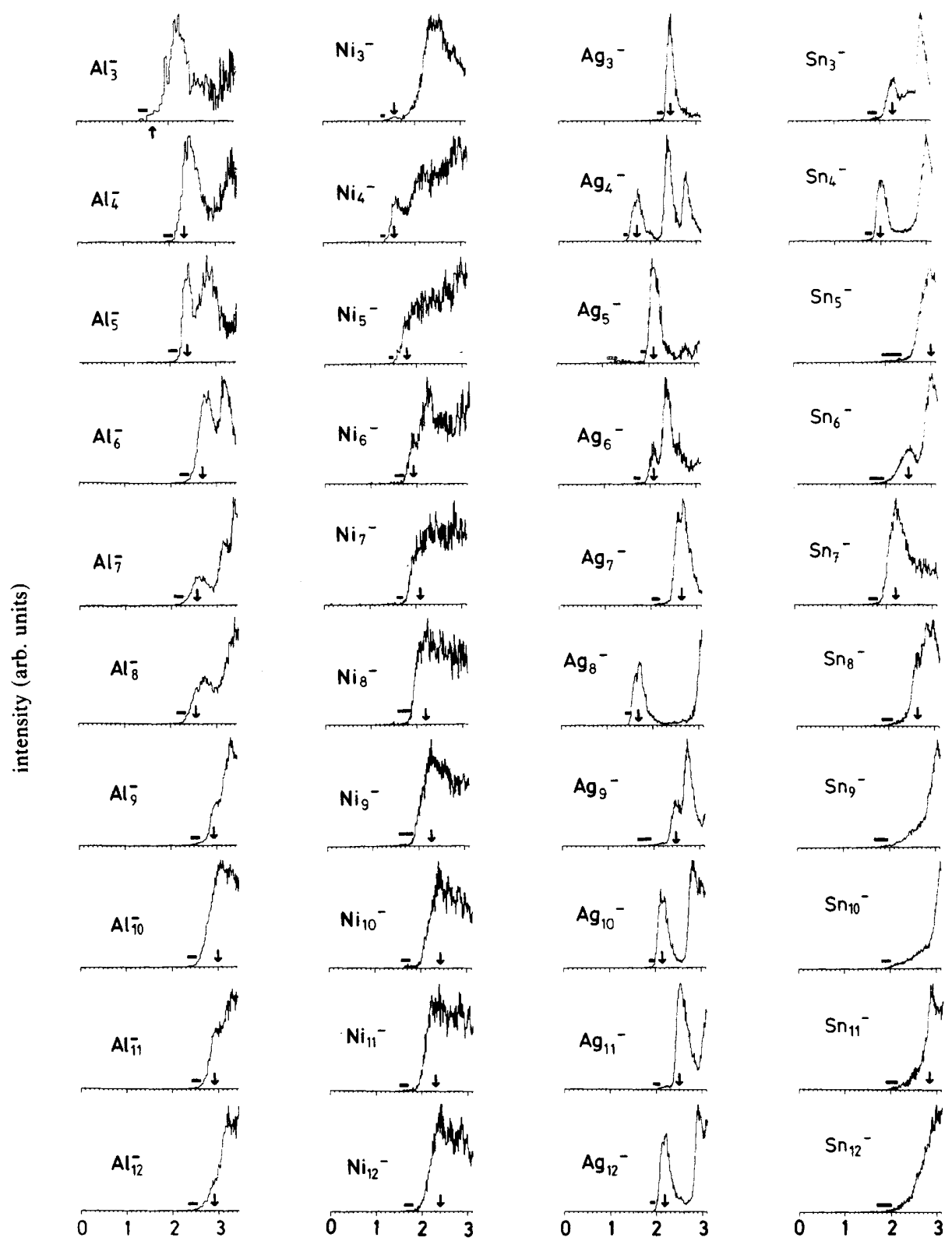

binding energy/eV

Fig. 4. Photoelectron spectra of $\mathrm{Al}_{n}^{-}(n=3-22), \mathrm{Ni}_{n}^{-}(n=3-18), \mathrm{Ag}_{n}^{-}(n=3-22)$ and $\mathrm{Sn}_{n}^{-}(n=3-22)$ at a detachment energy of $h \nu=3.68 \mathrm{eV}(337 \mathrm{~nm})$. The spectra are summed over $10^{4}-10^{5}$ shots with a background signal negligible on the scale shown. Photoelectron threshold energies as estimates of upper bounds of adiabatic electron affinities are marked by horizontal bars, the arrows indicate the positions of the first maxima. 


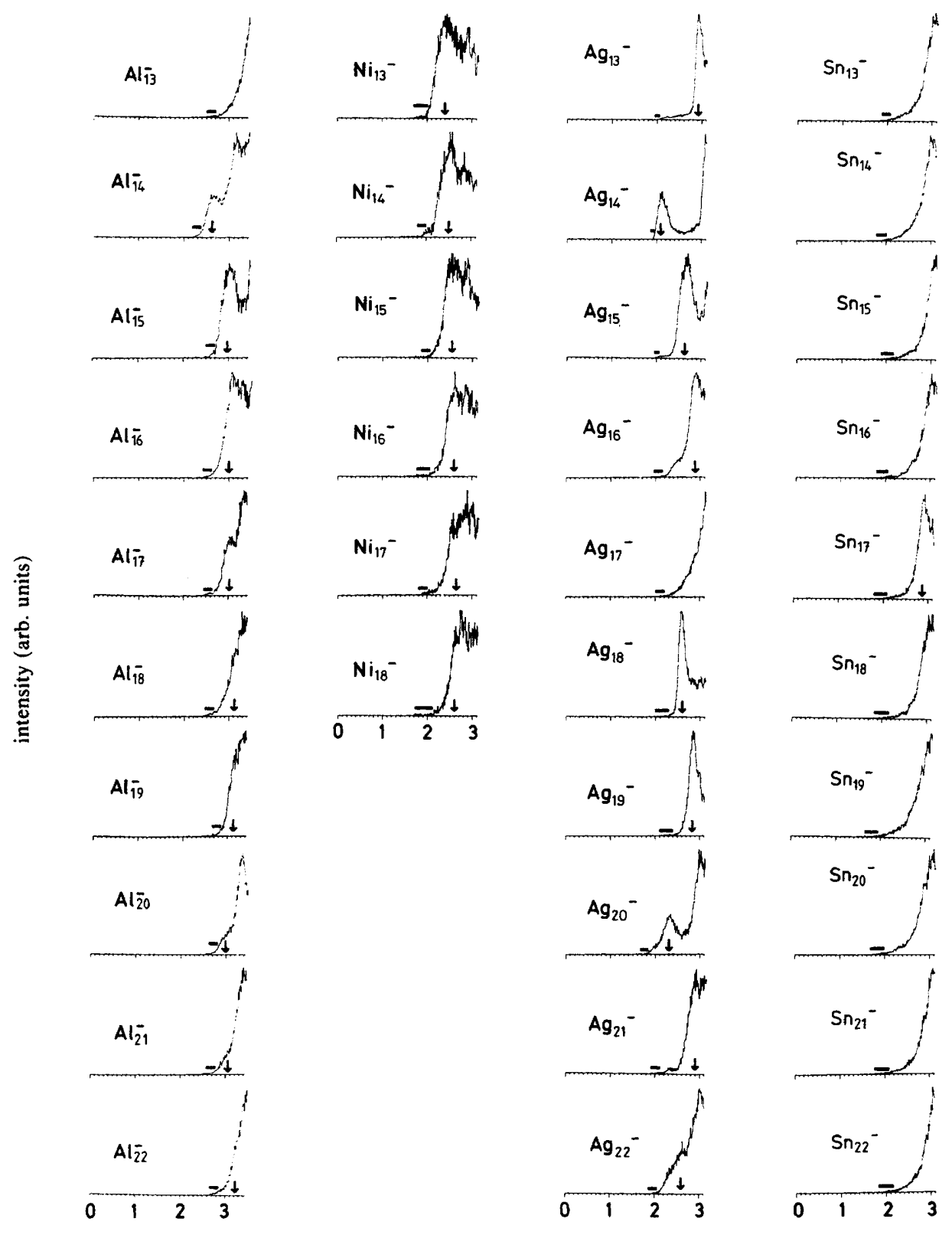

binding energy/eV

Fig. 4. Continued. 
are needed for cluster production, the mass peaks are further broadened, mainly due to space-charge effects.

For photodetachment, the mass-defined cluster-anion bunches are exposed to pulsed u.v. laser light $(h \nu=3.68 \mathrm{eV})$. The resulting p.e. energy spectra of aluminium $(n=3-22)$, nickel $(n=3-18)$, silver $(n=3-22)$ and tin $(n=3-22)$ are shown in fig. 4 . The rows contain spectra which belong to clusters of the same number of atoms, $n$. All cluster anions in this size range can be photodetached by $3.68 \mathrm{eV}$ light. Obviously each cluster possesses its own p.e. intensity profile, its characteristic 'fingerprint'. Not only do the p.e. thresholds vary strongly with $n$, but also the line positions. In some cases pronounced band gaps are seen, which may vanish completely when the cluster size increases by one or two additional atoms ( $c f$. e.g. $\left.\mathrm{Ag}_{n}, n=14-16\right)$. Other spectra $\left(\right.$ e.g. $\left.\mathrm{Ni}_{n}^{-}\right)$are nearly unstructured. In these studies, particular attention has been drawn to possible twophoton processes or cluster fragmentation upon photodetachment. ${ }^{23}$ There are features which appear in several spectra at the same p.e. energies: e.g. the strong-line in $\mathrm{Ag}_{6}^{-}$ appears at the position of the $\mathrm{Ag}_{3}^{-}$main peak, or the first peaks in the case of $\mathrm{Ag}_{10}^{-}$and $\mathrm{Ag}_{12}^{-}$correspond to the same electron binding energies as the small (low-energy) peak of $\mathrm{Ag}_{11}^{-}$. It is theoretically possible that such correspondences might originate from e.g. a two-step process in which the cluster undergoes photofragmentation first, and a second photon detaches the electron from the fragment anion. However, all the features remain unchanged when the laser intensity is lowered by as much as a factor of ten. Therefore, fragmentation and multiphoton effects can probably be excluded.

\section{Cluster-ion Production}

\section{Discussion}

The interaction of high-power laser radiation with materials is a matter of thorough investigation. $^{24}$ Surface ablation effects are of interest in the context of processes occurring in laser-induced thermal desorption, which has emerged as a new tool in the study of desorption dynamics at surfaces. The emission of positive and negative ions, on the other hand, is not understood so far. Some results favour a thermionic ion emission; ${ }^{25}$ we find, however, a non-thermal energy distribution when the ion's energies are analysed without using a carrier gas in the cluster source. ${ }^{26}$ In actual source operation, this 'superheated metal' or plasma is then entrained in the $\mathrm{He}$ or $\mathrm{Ne}$ carrier gas at a pressure of $c a$. $1 \mathrm{~atm} \dagger$ in the source channel. In $c a \cdot 10^{4}-10^{5}$ collisions it is rapidly quenched and cluster growth occurs. Cluster aggregation in the presence of $\mathrm{He}$ carrier gas has been calculated by Bernholc and Phillips. ${ }^{27}$ According to their model two limiting-growth regimes exist: the 'diffusion-controlled regime', where the sticking probability is large enough to assure cluster formation during each encounter, and the 'reaction-limited' regime, where many encounters are needed before cluster aggregation takes place.

In the reaction-limited regime clusters with high binding energy should be preferentially formed yielding a structured mass spectrum. Our cluster ion distributions ( $c f$. fig. 3) are essentially featureless, which holds true for positively and negatively charged clusters. Therefore, in terms of aggregation kinetics, all metal cluster ions and anions we studied so far (note that we do not apply any post-ionization) belong to the diffusion-controlled regime. In other words, the ( $n$-dependent) cluster's relative binding energy is not responsible for the abundances in these spectra.

The final shape of a mass spectrum is not only determined by the aggregation conditions but also by the cluster's vibronic excitation ('temperature') and by the timescale between ion production and detection. It is well known that mass spectra of sputtered (metal) cluster ions can be dominated by intensity anomalies: for example,

$\dagger 1 \mathrm{~atm}=101325 \mathrm{~Pa}$. 
sputtered $\mathrm{Al}_{n}^{-}$clusters have a huge intensity step beyond $\mathrm{Al}_{13}^{-}$, for $\mathrm{Al}_{n}^{+}$such steps are found at $n=7,14,23,29 .{ }^{17}$ Monovalent cluster ions show strong even-odd intensity alternations [for $\mathrm{Ag}_{n}$ see ref. (28), for $\mathrm{Cu}_{n}$ see ref. (28)-(30)], which are overlayed by pronounced intensity steps: in the case of monovalent negative clusters the steps appear at $n=7,19,33,39$ etc., for positive clusters at $n=9,21,35,41$ etc. On the other hand, the mass spectrum of sputtered nickel clusters, for example, has no irregularities. ${ }^{31} \mathrm{We}$ have shown ${ }^{29,32}$ that the ion distributions of sputtered clusters result of cascades of metastable decay processes of an unknown initial distribution. The decay rate constants are strongly dependent on the time windows in which the decompositions are observed $;^{33}$ therefore, the shapes of mass distributions depend also on the mass analysis method. Even jet-cooled (neutral) metal clusters tend to undergo decompositions upon nearthreshold photoionization. ${ }^{34}$ In contrast to these situations our mass spectra of jet-cooled cluster ions (see fig. 3) are unstructured, therefore proving that the cluster's internal energy is below the fragmentation energy.

We note that other authors ${ }^{35-37}$ have investigated (mainly semiconductor) cluster ions produced in a laser vaporization source, also without converting neutral clusters into ions via a secondary ionizing agent. They find strong fluctuations in the intensity distributions; these might have their origin either in a different source geometry, or in collision-induced fragmentation of the (accelerated) ions. In contrast, in our experiment the region between nozzle and skimmer is carefully shielded from electric fields in order not to disturb the supersonic expansion.

\section{Electronic Properties of Metal Clusters probed by Photoelectron Spectroscopy}

The photoelectron spectra as shown in fig. 4 result from transitions of the cluster anion's ground states to ground as well as to several excited neutral cluster states. When we treat p.e. emission in a simple molecular-orbital picture the total energy $h \nu$ of an absorbed photon is taken by a single electron, and the energy of this electron before excitation may be found simply by substracting $h \nu$ from the measured p.e. energy [see fig. 5(a)]. A more appropriate point of view is to consider the electron states of an $n$-electron system and compare them to those of the $(n-1)$-electron system left behind after the photoemission event. If $n$ is large, these states will not differ significantly since the electron potential does not change much when a single electron is removed. In systems with a small number of electrons, on the other hand, the removal of a single electron might result in a change of the electric field. The orbitals of the remaining electrons adjust to the new field, which may influence the chemical bonds and thus induce vibrational excitation [see fig. $5(b)$ ].

For the metal cluster anions discussed here the degree of geometrical change upon neutralization is not known, i.e. no information is yet available about the difference between vertical and adiabatic electron affinities. Therefore, the shapes of the p.e. intensity profiles near threshold might reflect the geometric rearrangement, the density of states, or a convolution of both. In the absence of a better definition we define the p.e. thresholds (given by horizontal bars in fig. 4) as upper limits of adiabatic electron affinities. Several electron spectra show non-sharp threshold behaviour so that uncertainties in the threshold energy determination are mainly physical in nature rather than instrumental. The onset uncertainties vary with the cluster size as marked by the bar widths in fig. 4 .

In order to describe the threshold $n$-dependence of all four cluster systems we apply a simple electrostatic model in which the image charge interaction between an electron and a conducting neutral sphere leads to the following prediction for the electron affinities $A_{\mathrm{e}}$ as function of the sphere's radius $R:^{38}$

$$
A_{\mathrm{e}}(R)=W F-\frac{5}{8}\left(e^{2} / R\right)
$$

For large $R, A_{\mathrm{e}}$ approaches the bulk work function $W F$. 


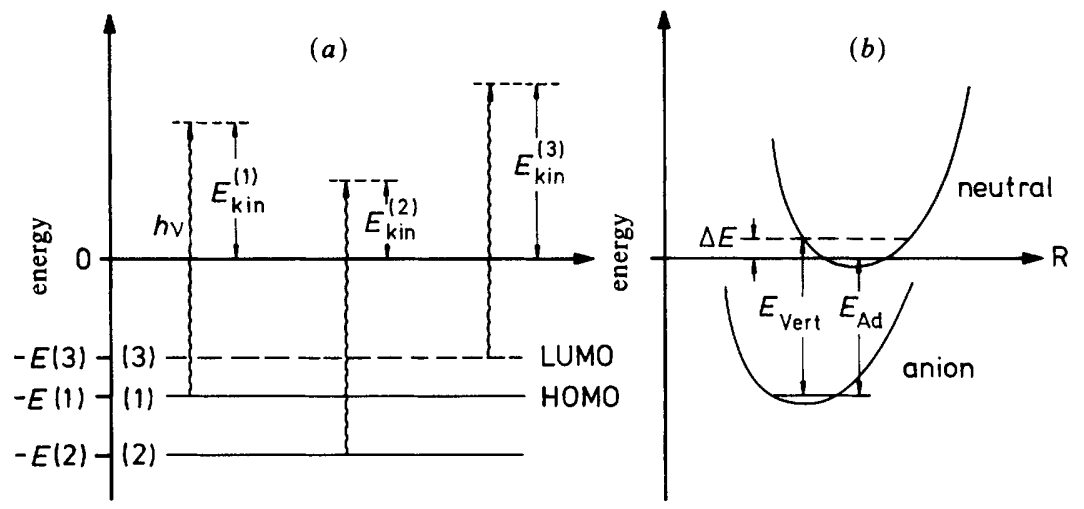

Fig. 5. (a) Simplified scheme of the photoejection process: Laser light at fixed energy $h \nu$ ionizes an electron from the HOMO (1) or from a deeper orbital (2). The electron energies, $E_{\text {kin }}$, are a direct measure of the orbital positions. In ground-state (cluster) anions the additional electron occupies the LUMO and can lead to a photoelectron of higher kinetic energy. (b) Schematic potential-energy curves of neutral and anionic molecules or small clusters. A vertical electronic transition $E_{\text {ver }}$ will produce vibrationally excited neutral species when the geometric structure (here the internuclear separation $R$ ) of the ground-state anion differs from that of the neutral. The energy difference $\Delta E$ between vertical excitation energy, $E_{\text {vert }}$, and the adiabatic electron binding energy, $E_{\text {ad }}$, will vibrationally excite the neutral cluster.

This classical spherical drop model is in rough agreement with observed $\mathrm{Cu}_{n}$ electron affinities when $R$ is given by

$$
R=r n^{1 / 3}+r_{0}
$$

with $r$ being the mean half distance between the atoms and $r_{0}$ a correction taking into account the 'spilling-out' of the electron density beyond the cluster radius. ${ }^{1}$ To linearize eqn (1) we plot $A_{\mathrm{e}}(R)-W F$ vs. $1 / R$, the $A_{\mathrm{e}}$ values being the measured p.e. threshold energies of fig. 4. In the absence of the exact cluster internuclear distances the respective bulk values $\left(r=r_{\text {bulk }}\right)$ are chosen; $r_{0}$ is taken to be the Bohr radius $a_{0}$. Fig. 6 compiles $A_{\mathrm{e}}(n)$ (p.e. threshold) as given by the spectra of fig. 4. The data can roughly be represented by straight lines. The classically expected behaviour [eqn (1)] is given by the dashed line. When using a similar representation of ionization potentials the data of numerous metal cluster systems were found to be approximated by a single straight line. ${ }^{39}$ For p.e. thresholds, however, such a simple fit is not possible; with a variation of $r$ within $80-100 \%$ of $r_{\text {bulk }}$, and different $r_{0}$ values $\left(r_{0}=0, \ldots, 2 a_{0}\right)$ the $\mathrm{Al}_{n}^{-}$and $\mathrm{Ag}_{n}^{-}$ data can be shifted to lie near the dashed line. The values of $\mathrm{Ni}_{n}^{-}$and $\mathrm{Sn}_{n}^{-}$, however, remain far from the classical model, as well as those of lead cluster anions. ${ }^{40}$ Apparently, the spherical-drop model describes the p.e. thresholds of some cluster systems. In other cases, and especially those where non-ideal electron delocalisation is expected $\left(\mathrm{Sn}_{n}\right.$, $\mathrm{Ni}_{n}$ etc.), the model fails. Corrections including cluster-specific polarizabilities might be successful.

A number of characteristic deviations from the linear threshold dependence on $1 / R$ occur which can in part be explained by 'jellium' calculations: ${ }^{41}$ In this model the atomic valence electrons are filled into a potential made up by the homogeneously distributed positive charge of cores. Enhanced binding energies are obtained for closed-shell structures. The same model works for alkali-metal-like and other simple metals like aluminium, where the bulk properties can be described adequately by the nearly free electron model, and the details of the screened ionic potential introduce only minor 


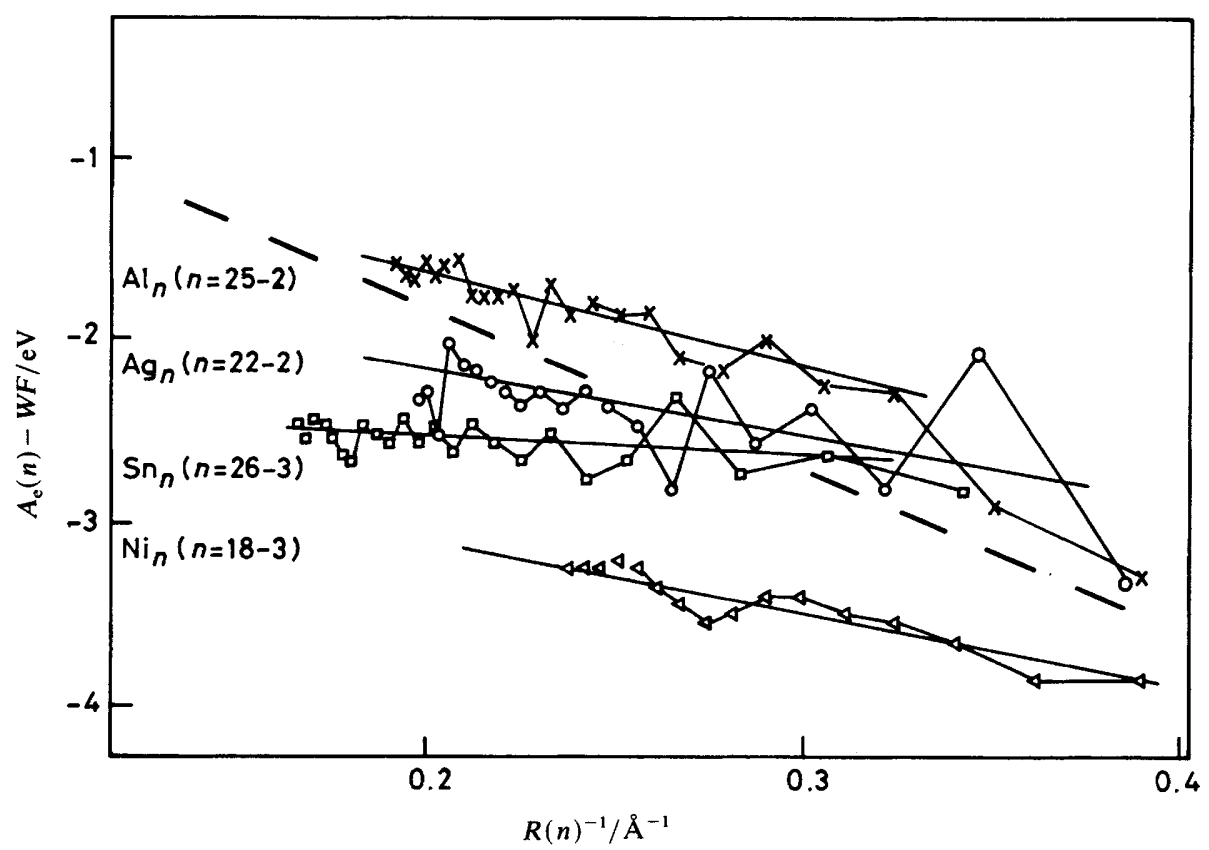

Fig. 6. Difference between experimental electron affinities (p.e. thresholds) and bulk work function based on the spherical-drop model according to eqn (1). $\times, \mathrm{Al}_{n}(n=25-2) ; \mathrm{O}, \mathrm{Ag}_{n}(n=22-2)$; $\square, \mathrm{Sn}_{n}(n=26-3) ; \triangle, \mathrm{Ni}_{n}(n=18-3)$. For the cluster radii determination [eqn (2)] bulk internuclear distances have been chosen. The image charge potential between an electron and a classical conducting sphere with radius $R$ yields the dashed line.

effects. $^{42}$ Aluminium is trivalent and the calculated shell closings are rarely integer multiples of three; therefore, the effects might not be so distinct as in the case of, e.g. silver. Within the scope of this experiment only one exact aluminium shell closing appears at $\mathrm{Al}_{13}^{-}$(40 electrons). The corresponding p.e. spectrum shows a slightly enhanced value for the electron threshold and a substantial shift of the first maximum to higher binding energy (beyond the energy accesible). The spectral difference from $\mathrm{Al}_{14}^{-}$is pronounced, a clear sign of a shell effect. For silver clusters the main shell spacings are expected at $n=8,18$ and 20. Pronounced threshold minima are observed at $n=8$ and 20 .

Beyond the comparatively simple jellium model, elaborate quantum-chemical calculations have been performed on the electronic properties of small metal clusters, taking into account the geometric arrangements of their ionic cores. Aluminium clusters are characterized by a number of low-lying electronic excited states as a result of the different spin multiplicities and/or the $s^{2} p^{1}$ atomic valence configuration. ${ }^{43-46}$ Even for the dimer ca. 20 singlet and triplet states might be combined from two ground-state Al atoms. All of these states are expected to lie within 1-4 eV of the molecular ground state. This rich array of states breaks into manifolds of $\Sigma, \Pi$, and $\Delta$ symmetry. Going to higher $n$, the complexity will not be reduced; therefore, it is not surprising that the theoretical studies are mostly in disagreement as far as the state ordering is concerned.

For a further discussion of $\mathrm{Al}_{n}^{-}$p.e. spectra we refer to ref. (15).

In nickel clusters the two nearly degenerate lowest-lying states of the $\mathrm{Ni}$ atom, ${ }^{3} \mathrm{D}$ and ${ }^{3} \mathrm{~F}$, produce numerous low-lying states even in small clusters. The dimer, e.g. has 30 electronic states within an energy range of $0.6 \mathrm{eV}$, and the state density of the trimer 
is calculated to be even higher. ${ }^{47}$ Correspondingly, the intensity profiles of all $\mathrm{Ni}_{n}$ p.e. spectra are very dense; with the exception of $\mathrm{Ni}_{6}$ no distinct bands are seen. Therefore, p.e. intensity profiles of nickel clusters look like typical bulk metal p.e. spectra near the Fermi level.

The bonding in silver clusters is determined by the 5 s electron of the alkali-metal-like atom. The interaction of the $d$ orbitals should be low so that (as in the case of copper and alkali-metal clusters) a pronounced even/odd behaviour governs the electronic structure. Alternations in photoionization profiles of alkali-metal clusters, ${ }^{48,49}$ in unimolecular decay rates and fragmentation cross sections of copper clusters, ${ }^{17,29}$ and in photofragmentation of silver clusters ${ }^{50}$ have been observed. The strong similarity of alkali-metal-like clusters is especially obvious when the silver photoelectron spectra (fig. 4) are compared to those of copper cluster anions: ${ }^{1,2}$ at a first sight the spectral shapes are identical in the region of overlap. In some cases even the energetic positions of the $\mathrm{Ag}_{n}$ and $\mathrm{Cu}_{n}$ bands are the same. Recent measurements of alkali-metal-cluster photoelectron spectra ${ }^{4}$ show in part a qualitatively similar band structure of potassium anions when compared with those of silver and copper clusters. The absolute energetic positions, however, are shifted to lower electron binding energies.

For tin clusters no theory is yet available, nor are experimental studies which could be compared to our results. Bulk tin is known to be at the border between semiconductor and metal with a nearly zero band gap. It is unknown whether tin clusters tend to have metallic or non-metallic properties. From the $R$-dependence of the $\mathrm{Sn}$ p.e. threshold values (fig. 6) we conclude that $\mathrm{Sn}$ clusters do not behave like metal droplets. The pronounced HOMO-LUMO gap of $\mathrm{Sn}_{4}$, on the other hand, could correspond to a shell closing ( 8 electrons). Other hints of shell effects (e.g. 20 electrons) are missing.

\section{Conclusion}

Gas-phase metal-cluster anions have been prepared by laser vaporization techniques and studied by photoelectron spectroscopy. All mass spectra show uniform broad intensity distributions. This indicates that relative cluster binding energies do not determine the aggregation process when the ions grow in the source channel and undergo jet expansion. We conclude that the clusters thus produced are cold, having internal energies not sufficient for fragmentation. Using a photon energy $h \nu=3.68 \mathrm{eV}$, we have studied photodetachment from $\mathrm{Al}_{n}^{-}, \mathrm{Ni}_{n}^{-}, \mathrm{Ag}_{n}^{-}$and $\mathrm{Sn}_{n}^{-}, n \leqslant 22$. The photoelectron spectra are strongly size- and material-dependent and allow a glimpse into the cluster's density of states: the spectra of, e.g. nickel-cluster anions, are very dense; silver-cluster spectra, on the other hand, show well separated peaks with band gaps which might exceed $1 \mathrm{eV}$. Theoretical data are available only in some cases. Simple models still continue to play an important role in the explanation of such photoelectron spectra.

We thank H. R. Siekmann for his help in the early stages of the experiment. This work was supported by the Deutsche Forschungsgemeinschaft (DFG).

\section{References}

1 D. G. Leopold, J. H. Ho and W. C. Lineberger, J. Chem. Phys,, 1987, 86, 1715.

2 O. Cheshnovsky, P. J. Brucat, S. Yang, C. L. Pettiette, J. J. Craycraft and R. E. Smalley, in The Physics and Chemistry of Small Clusters, NATO ASI Series B, 1987, 158, 1.

3 G. Ganteför, K. H. Meiwes-Broer and H. O. Lutz, Phys. Rev. A, 1988, 37, 2716.

$4 \mathrm{~K}$. H. Bowen, to be published.

5 Cf. Elemental and Molecular Clusters, Springer Series in Materials Science (Springer-Verlag, Berlin, 1988), vol. 6.

6 J. Farges, M. F. de Feraudy, B. Raoult and G. Torchet, J. Chem. Phys., 1986, 84, 3491.

7 M. F. Jarrold and J. E. Bower, J. Chem. Phys., 1986, 85, 5373.

8 W. D. Reents Jr, A. M. Mujsce, V. E. Bondybey and M. L. Mandich, J. Chem. Phys., 1987, 86, 5568. 
9 A. W. Castlemann Jr and R. G. Keesee, Z. Phys. D, 1986, 3, 167.

10 M. M. Kappes, M. Schär, P. Radi and E. Schumacher, J. Chem. Phys., 1986, 84, 1863.

11 E. A. Rohlfing, D. M. Cox, A. Kaldor and K. H. Johnson, J. Chem. Phys., 1984, 81, 3846.

12 C. Brechignac and Ph. Cahusac, Z. Phys. D, 1986, 3, 121.

13 T. Bergmann, H. Limberger and T. P. Martin, Phys. Rev. Lett., 1988, 60, 1767.

14 L. Cordis, G. Ganteför, J. Heßlich and A. Ding, Z. Phys. D, 1986, 3, 323.

15 G. Ganteför, M. Gausa, K. H. Meiwes-Broer and H. O. Lutz, Z. Phys. D, 1988, 9, 253.

16 V. E. Bondybey and J. H. English, J. Chem. Phys., 1981, 74, 6978; T. G. Dietz, M. A. Duncan, D. E. Powers and R. E. Smalley, J. Chem. Phys., 1981, 74, 6511.

17 W. Begemann, S. Dreihöfer, G. Ganteför, H. R. Siekmann, K. H. Meiwes-Broer and H. O. Lutz, in Elemental and Molecular Clusters, Springer Series on Material Science (Springer-Verlag, Berlin, 1988), vol. 6 , p. 230.

18 P. Kruit and F. H. Read, J. Phys. E, 1983, 16, 313.

19 H. Hotop and W. C. Lineberger, J. Phys. Chem. Ref. Data, 1975, 4, 539.

20 V. A. Esaulov, Ann. Phys. Fr., 1986, 11, 493.

21 C. F. Moore, Atomic Energy Levels, Natl. Bur. Stand., Ref. Data Ser. 35 (Natl Bur. Stand., Washington D.C., 1971).

22 W. C: Wiley and I. H. McLaren, Rev. Sci. Instr., 1955, 26, 1150.

23 P. Fayet, W. A. Saunders and L. Wöste, to be published.

24 J. F. Ready, in Effects of High-power Laser Radiation (Academic Press, London, 1971).

25 R. Viswanathan and 1. Husla, in Laser Processing and Diagnostics, Springer Series in Chemical Physics (Springer-Verlag, Berlin, 1984), vol. 39.

26 H. R. Siekmann, G. Ganteför, M. Gausa, K. H. Meiwes-Broer and H. O. Lutz, to be published.

27 J. Bernholc and J. C. Phillips, J. Chem. Phys., 1986, 85, 3258.

28 I. Katakuse, T. Ichihara, Y. Fujita, T. Matsuo, T. Sakurai, and H. Matsuda, Int. J. Mass Spectrom. Ion Process., 1985, 67, 229.

29 W. Begemann, S. Dreihöfer, K. H. Meiwes-Broer and H. O. Lutz, Z. Phys. D, 1986, 3, 183.

30 W. Begemann, S. Dreihöfer, K. H. Meiwes-Broer, H. O. Lutz, in The Physics and Chemistry of Small Clusters, NATO ASI Series B, 1987, 158, 269.

31 P. Fayet, L. Wöste, Spectrosc. Int. J., 1984, 3, 91.

32 W. Begemann, K. H. Meiwes-Broer and H. O. Lutz, Phys. Rev. Lett., 1986, 56, 2248.

33 W. Begemann, K-H. Miewes-Broer and H. O. Lutz, J. Phys. (Paris), to be published.

$34 \mathrm{C}$. Brechignac and $\mathrm{Ph}$. Cahuzac, to be published.

35 M. E. Geusic, R. R. Freeman and M. A. Duncan, J. Chem. Phys., 1988, 88, 163.

36 L. A. Bloomfield, M. E. Geusic, R. R. Freeman and W. L. Brown, Chem. Phys. Lett., 1985, $121,33$.

37 Y. Liu, Q-L. Zhang, F. K. Tittel, R. F. Curl and R. E. Smalley, J. Chem. Phys., 1986, 85, 7434.

38 D. M. Wood, Phys. Rev. Lett., 1981, 46, 749.

39 E. Schumacher and M. M. Kappes, in Large Finite Systems, ed. J. Jortner and A. Pullman (D. Reidel, Dordrecht, 1987).

40 G. Ganteför, M. Gausa, K-H. Miewes-Boer and H. O. Lutz, Z. Phys. D, 1988, to be published.

41 (a) J. L. Martins, R. Car and J. Buttet, Surf. Sci., 1981, 106, 265; (b) W. Eckardt, Phys. Rev. B, 1984, 29, 1558; (c) W. Knight, K. Clemenger, W. de Heer, W. Saunders, M. Chou and M. Cohen, Phys. Rev. Lett., 1984, 52, 2141

42 M. Y. Chou and M. L. Cohen, Phys. Rev. Lett. A, 1986, 113, 420.

43 T. H. Upton, Phys. Rev. Lett., 1986, 56, 2168; J. Chem. Phys., 1987, 86, 7054.

44 G. Paccinioni, J. Koutecky, Ber. Bunsenges. Phys. Chem., 1984, 88, 242.

45 L. G. M. Pettersson, C. W. Bauschlichter Jr and T. Halicioglu, J. Chem. Phys., 1987, 87, 2205.

46 K. Hermann, H. J. Hass and P. S. Bagus, Z. Phys. D, 1986, 3, 159.

47 1. Shim, K. A. Gingerich, in The Physics and Chemistry of Small Clusters, NATO ASI Series B, 1987, $158,523$.

48 M. M. Kappes, M. Schär, U. Röthlisberger, C. Yeretzian and E. Schumacher, Chem. Phys. Lett., 1988, $143,251$.

49 C. Brechignac and Ph. Cahuzac, Chem. Phys. Lett., 1985, 117, 365.

50 P. Fayet and L. Wöste, Z. Phys. D, 1986, 3, 177. 